Erschienen in: Deutsche Sprache, 37 (2009), H. 1, S. 65-82

Kristel Proost

\title{
Bedeutung und Standardinterpretation von Äußerungen mit negierten negativ-bewertenden Adjektiven
}

\begin{abstract}
Thema dieses Beitrags ist der Unterschied zwischen der Bedeutung und der Standard- oder ,Default“-Interpretation von Äußerungen mit negierten lexikalischen bzw. un-präfigierten Antonymen graduierbarer Adjektive wie intelligent (z.B. X ist nicht dumm vs. $X$ ist nicht unintelligent). Ausgehend von der Darstellung der Bedeutung und der Standardinterpretation der entsprechenden nicht-negierten Formen dieser Äußerungen (z.B. $X$ ist dumm vs. $X$ ist unintelligent) wird zunächst gezeigt, dass Äußerungen wie $X$ ist nicht dumm und $X$ ist nicht unintelligent sich im Hinblick auf das, was mit ihnen kodiert ist, unterscheiden: Äußerungen mit negierten lexikalischen Antonymen ( $X$ ist nicht dumm) umfassen sowohl den positiven als auch den neutralen Mittelbereich der jeweils relevanten Skala, solche mit negierten un-präfigierten Antonymen ( $X$ ist nicht unintelligent) hingegen nur den positiven Bereich. Die beiden Typen von Äußerungen unterscheiden sich aber auch im Hinblick auf ihre Standardinterpretation: Obwohl sie beide überlicherweise als , eher $\mathrm{X}^{\star}$ oder ,ziemlich $\mathrm{X}^{\star}$ (z.B. ,eher intelligent ${ }^{\star}$ oder ,ziemlich intelligent ${ }^{\star}$ ) interpretiert werden, wird die mit den negierten, un-präfigierten Formen ausgedrückte Bewertung von Muttersprachlern (des Deutschen) häufig als positiver eingeschätzt als die Bewertung, die mit den negierten nicht-abgeleiteten Formen ausgedrückt wird.

This contribution deals with the difference between the meaning and the standard or default interpretation of utterances containing negated lexical vs. morphological antonyms of gradable adjectives such as intelligent (e.g. $X$ is not stupid vs. $X$ is not unintelligent). Starting from the description of the meaning and the standard interpretation of the positive equivalents of these utterances ( $X$ is stupid vs. $X$ is unintelligent), utterances like $X$ is not stupid and $X$ is not unintelligent are first shown to differ with respect to what they code: Utterances with negated lexical antonyms ( $X$ is not stupid) are used to refer to the positive as well as the neutral middle area of the relevant scale, while such with negated morphological antonyms ( $X$ is not unintelligent) may only be used to refer to the positive section of that scale. Apart from being different with respect to what they code, utterances like $X$ is not stupid and $X$ is not unintelligent also differ regarding their standard interpretation: both are interpreted by default as ,rather $X^{\star}$ or , fairly $X^{\text {‘ }}$ (e.g. as ,rather intelligent or ,fairly intelligent'), but the evaluation expressed by the negated morphological form is frequently judged by native speakers (of German) to be more positive than the one expressed by the negated lexical form.
\end{abstract}

\section{Einleitung}

Thema dieses Beitrags ist der Unterschied zwischen der Bedeutung und der Standardinterpretation von Äußerungen mit negierten lexikalischen bzw. un-präfigierten Antonymen graduierbarer Adjektive. Beispiele von Adjektiven, die sowohl ein lexikalisches als auch ein morphologisch verwandtes Antonym haben, sind intelligent und schön, für die es die Antonyme unintelligent und dumm bzw. unschön und hässlich gibt. Äußerungen mit den negierten Formen dieser beiden Varianten (Er ist nicht dumm vs. Er ist nicht unintelligent) werden verglichen im Hinblick auf das, was mit ihnen kodiert ist, und das, was mit ihnen default- oder standardmäßig implikatiert ist.

Statistische Untersuchungen $\mathrm{zu}$ den Gegensatzrelationen deutscher Adjektive haben gezeigt, dass die Zahl der morphologisch nicht-verwandten Antonyme die Zahl derer, die durch un-Präfigierung gebildet sind, bei weitem übersteigt (vgl. Rachidi 1989, S. 189192). Obwohl es insgesamt weitaus mehr Adjektive gibt, die entweder nur ein morphologisch verwandtes oder nur ein morphologisch nicht-verwandtes Antonym haben, als solche, die beide Typen von Antonymen haben, sei die Zahl der Letzteren dennoch nicht 
gering (vgl. ebd., S. 190). Zu den Adjektiven, die beide Typen von Antonymen haben, gehören viele Bewertungsprädikate. Beispiele sind gut-schlecht/ungut, glücklich-traurig/ unglücklich, froh-traurig/unfroh, spannend-langweilig/unspannend, ordentlich-schlampig/unordentlich, klug-dumm/unklug und die bereits erwähnten Paare intelligent-dumm/ unintelligent und schön-hässlich/unschön. Ein Beispiel eines nicht-bewertenden Adjektivs, das sowohl ein lexikalisches als auch ein un-präfigiertes Antonym hat, ist gesund, für das es die Antonyme krank und ungesund gibt.

Eine Untersuchung von Äußerungen mit negierten graduierbaren Adjektiven setzt voraus, dass geklärt ist, was mit den nicht-negierten Formen dieser Adjektive (z.B. unintelligent oder dumm) kodiert bzw. implikatiert ist: Zum kodierten Bereich von nicht unintelligent oder nicht dumm gehören alle Fälle, in denen jemand oder etwas nicht als unintelligent bzw. dumm beurteilt wird. Es wird daher zunächst auf die Bedeutung von Äußerungen wie $X$ ist dumm und $X$ ist unintelligent eingegangen, womit sich automatisch die Frage erhebt, ob diese äquivalent sind mit Äußerungen wie $X$ ist nicht intelligent. Ausgehend von den Überlegungen zur Bedeutung von Äußerungen wie $X$ ist unintelligent und $X$ ist dumm wird diskutiert, was mit den negierten Formen dieser Äußerungen, $X$ ist nicht dumm und $X$ ist nicht unintelligent, kodiert bzw. implikatiert ist, und ob sich diese beiden Typen von Äußerungen diesbezüglich voneinander unterscheiden.

\section{Zum Begriff der Standardinterpretation}

Die Unterscheidung von dem, was mit einer Äußerung kodiert ist, und dem, was mit ihr als Standardinterpretation implikatiert ist, geht auf die Unterscheidung von Sagen und Meinen zurück, die bereits im 4. Jhd. von den Rhetorikern Servius und Donatus zur Erklärung von Litotes, einer rhetorischen Figur der Untertreibung, herangezogen und 1975 von Grice in „Logic and Conversation“" wieder aufgegriffen wurde (vgl. Horn 2004, S. 3). Nach Grice (1975) setzt sich die Gesamtbedeutung einer Äußerung zusammen aus dem, was mit ihr gesagt ist, und dem, was mit ihr gemeint oder implikatiert ist. Das Gesagte umfasst neben dem, was wörtlich gesagt ist, auch alle Arten von semantischen Inferenzen (vgl. Harras 2003, S. 52 \& 2004, S. 238). Das Gemeinte oder Implikatierte besteht aus dem, was mit einer Äußerung konventional implikatiert ist, im Wesentlichen den Präsuppositionen, und dem, was mit ihr konversational implikatiert ist, den Implikaturen. Präsuppositionen tragen zum Wahrheitswert einer Äußerung bei; Implikaturen tun dies nicht (vgl. Harras 2004, S. 238; Horn 2004, S. 3). Die wahrheitswertrelevanten Anteile der Gesamtbedeutung einer Äußerung, das was mit ihr gesagt und das, was mit ihr konventional implikatiert ist, sind nach Levinson sprachlich kodiert. Das konversational Implikatierte sei hingegen nicht sprachlich kodiert, sondern werde aufgrund von Annahmen über die Rationalität sprachlichen Handelns inferiert (vgl. Levinson 2000, S. 14).

Grice unterscheidet zwei Arten von konversationalen Implikaturen: generalisierte und partikulare (vgl. Grice 1975, S. 56-58). Letztere sind situationsspezifisch und damit auf jeweils konkrete Vorkommen von Äußerungen bezogen. Generalisierte Implikaturen unterscheiden sich darin von partikularen, dass sie situationsunabhängig sind. Sie sind nicht auf konkrete Äußerungsexemplare, sondern auf bestimmte Typen von Äußerungen bezogen (vgl. Levinson 2000, S. 22-23). 
Der Unterschied zwischen generalisierten und partikularen Implikaturen kann durch das folgende Beispiel aus Levinson (2000, S. 16-17) verdeutlicht werden („PKI“ steht für ,partikulare konversationale Implikatur" und „GKI“ für ,generalisierte konversationale Implikatur'):

(1) Situation 1:

A: Wieviel Uhr ist es?

B: Einige Gäste sind schon gegangen.

PKI: Es ist schon spät.

GKI: Nicht alle Gäste sind gegangen.

(2) Situation 2:

A: Wo ist Peter?

B: Einige Gäste sind schon gegangen.

PKI 1: Vielleicht ist Peter schon gegangen.

PKI 2: Vielleicht ist Peter noch da.

GKI: Nicht alle Gäste sind gegangen.

Beispiele (1) und (2) zeigen, dass die Äußerung Einige Gäste sind schon gegangen unabhängig von der Situation, in der sie gemacht wird, die Interpretation, Nicht alle Gäste sind gegangen' implikatiert. Immer, wenn jemand eine Äußerung des Typs ,Einige $X$ sind $Y^{\text {‘ }}$ macht, wird diese als ,Nicht alle $\mathrm{X}$ sind $\mathrm{Y}^{6}$ interpretiert. Die generalisierte Implikatur ,Nicht alle $X$ sind $Y^{\prime}$ ist die Standardinterpretation von Äußerungen des Typs, Einige $X$ sind $\mathrm{Y}^{6}$. Standardinterpretationen wie diese haben den Status einer Präsumption, d.h. einer Annahme, die solange als wahr gilt, bis ihr Gegenteil erwiesen ist.

Insgesamt setzt sich die Gesamtbedeutung einer Äußerung aus den folgenden Anteilen zusammen:

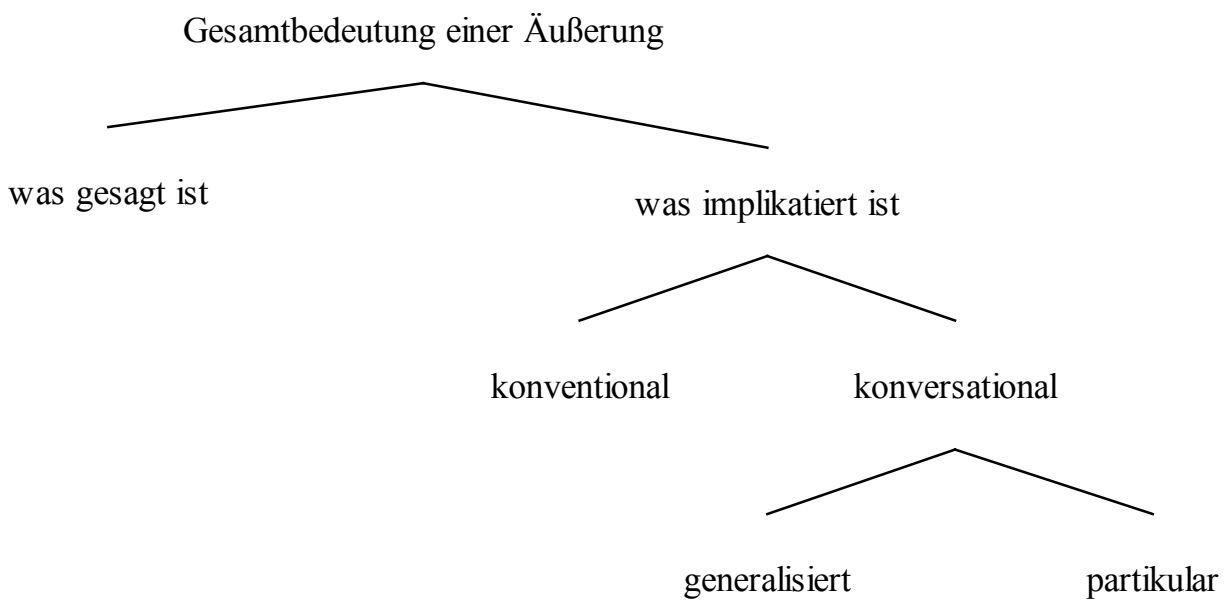

Abb. 1: Anteile der Gesamtbedeutung einer Äußerung (nach Levinson 2000, S. 13)

Da generalisierte Implikaturen situationsunabhängig sind, erhebt sich die Frage nach ihrer Abgrenzung von dem, was mit einer Äußerung kodiert ist. In Bezug auf die Äußerung Einige Gäste sind schon gegangen könnte man beispielsweise behaupten, dass ,nicht alle“ 
eine semantische Inferenz aus einige und damit Teil der lexikalischen oder konventionalen Bedeutung dieses Wortes ist. Gegen diese Auffassung spricht allerdings, dass semantische Inferenzen im Unterschied zu Implikaturen nicht getilgt oder verstärkt werden können. Wie die Beispiele in (3) und (4) zeigen, erlaubt die Interpretation von einige als ,nicht alle“ sowohl Tilgung als auch Verstärkung (vgl. Levinson 2000, S. 81):

(3) Einige, aber nicht alle Gäste sind schon gegangen.

(4) Einige, wenn nicht alle Gäste sind schon gegangen.

Nach Harras (2004, S. 240) gibt es außerdem Kontextbeschränkungen für den Gebrauch von nicht alle anstelle von einige:

(5) Der Kindergarten bleibt morgen geschlossen, weil einige/'nicht alle Kinder krank sind. (Beispiel aus Harras 2004, S. 240)

Da ,nicht alle“ sowohl getilgt als auch verstärkt werden kann und einige nicht in jedem beliebigen Kontext durch ,nicht alle` ersetzt werden kann, stellt ,nicht alle' keine semantische Inferenz, sondern eine generalisierte Implikatur aus einige dar; ,nicht alle‘ ist mit einige nicht kodiert, sondern implikatiert.

Die Unterscheidung von dem, was mit einer Äußerung kodiert ist und dem, was mit ihr default- oder standardmäßig implikatiert ist, ist auch für die Interpretation von Äußerungen mit negierten positiv-bewertenden Adjektiven relevant.

\section{3. Äußerungen mit negierten positiv-bewertenden Adjektiven}

Bereits Lyons hat darauf hingewiesen, dass negierte graduierbare Adjektive wie nicht gut üblicherweise als das konträre Antonym des entsprechenden Adjektivs (hier schlecht) verstanden werden. Wenn ein Sprecher einen anderen Sprecher beispielweise fragt, ob eine dritte Person X ein guter Schachspieler ist, und der zweite Sprecher diese Frage mit Nein beantwortet, wird diese Antwort normalerweise so interpretiert, dass der Sprecher, der sie geäußert hat, die betreffende Person als einen schlechten Schachspieler einschätzt (vgl. Lyons 1977, S. 278). Horn spricht in diesem Zusammenhang von „,contraries in contradictory cloathing“" (vgl. Horn 1989, S. 332): Das konträre Antonym (schlecht) wird als kontradiktorisch (nicht gut) getarnt. Gemeint ist ,X ist ein schlechter Schachspieler", gesagt wird aber nur, dass $\mathrm{X}$ kein guter Schachspieler ist.

Semantisch gesehen sind gut und schlecht konträre Antonyme. Das zeigt sich in den folgenden, für Kontrarietät typischen Implikationsverhältnissen: $X$ ist gut impliziert ,X ist nicht schlecht', und $X$ ist schlecht impliziert ,X ist nicht gut ${ }^{6}$, aber $X$ ist nicht gut impliziert nicht , $\mathrm{X}$ ist schlecht' und $X$ ist nicht schlecht impliziert nicht , $\mathrm{X}$ ist gut ${ }^{6}$. Das liegt daran, dass die Skala der Möglichkeiten, auf die mit konträren Antonymen Bezug genommen wird, einen neutralen Mittelbereich enthält, der weder vom einen noch vom anderen Ausdruck eines konträren Antonymenpaares bezeichnet wird (vgl. Lyons 1977, S. 272; Cruse 1986, S. 204; Lehrer 2002, S. 502; Löbner 2003, S. 124). Die Bewertungsskala ,gutschlecht' umfasst beispielsweise einen neutralen Mittelbereich, der alle Fälle erfasst, in denen etwas weder als gut noch als schlecht beurteilt wird.

Obwohl Äußerungen wie $X$ ist nicht gut logisch gesehen bedeuten, dass X entweder schlecht oder weder gut noch schlecht ist, werden sie üblicherweise als , $X$ ist schlecht ${ }^{\text {" }}$ interpretiert; ,X ist schlecht' ist die Standard- oder „Default“-Interpretation von Äuße- 
rungen wie $X$ ist nicht gut. Die Standardinterpretation von Äußerungen wie $X$ ist nicht gut als ,X ist schlecht' ist ein Beispiel von negativer Verstärkung: Sie schließt die schwächere Interpretation, weder gut noch schlecht', die logisch ebenfalls möglich ist, aus, wodurch der Intensivierungsgrad, der durch das negative Prädikat ausgedrückt wird, verstärkt wird (vgl. Abb. 2).

SCHLECHT

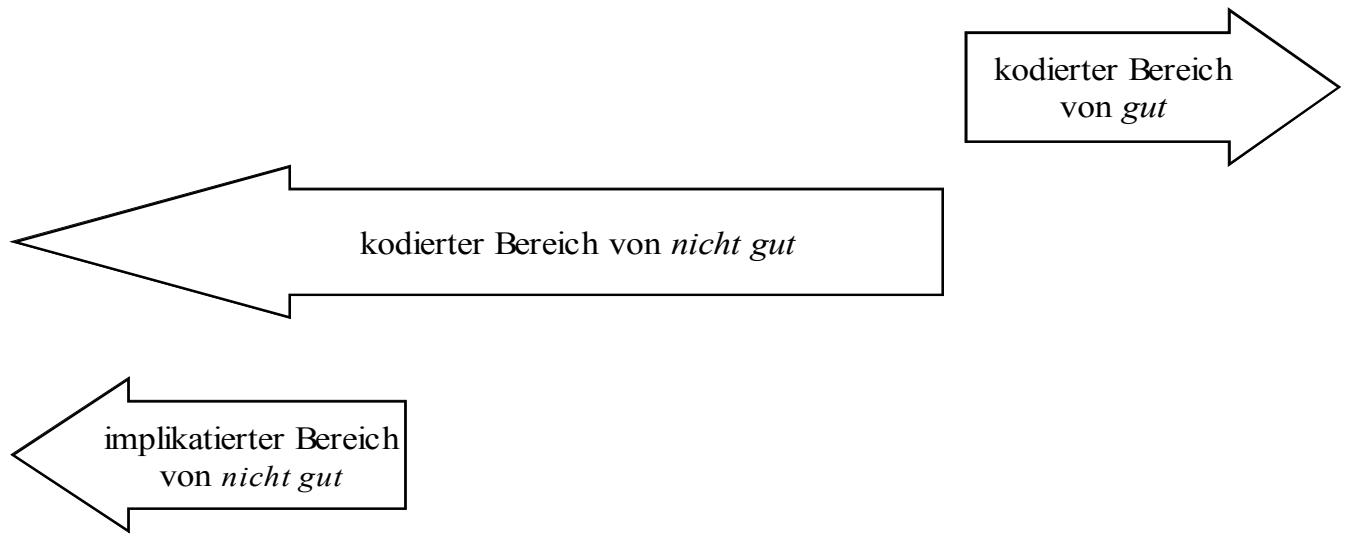

Abb. 2: Kodierter und implikatierter Bereich von nicht gut

Negative Verstärkung tritt auf, wenn der unmarkierte bzw. positiv-bewertende Ausdruck eines Paares konträrer Antonyme negiert wird: nicht gut wird dann als ,schlecht', nicht glücklich als ,unglücklich', nicht optimistisch als ,pessimistisch ' usw. interpretiert (vgl. Horn 1989, S. 333-335; Levinson 2000, S. 128; Blutner 2004, S. 500).

Auslöser des Effekts der negativen Verstärkung bei der Interpretation von Äußerungen mit negierten, graduierbaren Adjektiven ist nach Lyons eine generelle Präferenz für binäre Klassifikationen:

For most practical purposes we can usually get along quite well by describing things, in a first approximation as it were, in terms of a yes/no classification, according to which things are either good or bad, big or small, etc. (relevant to some relevant norm). If we deny that something is good or assert that it is not good without qualifying our statements in any way or supplying further information relevant to this dichotomous yes/no classification, it is reasonable for the other participants to assume that we are satisfied with a first approximation in terms of which gradable antonyms are interpretable as contradictories. ... If the speaker did not wish to be committed to the implication, he could have been expected to make it clear that a first approximation was insufficiently precise, by saying, for example, $X$ is not good, but he's not bad either: he's fairly/pretty good/just about average. (Lyons 1977, S. 278)

Die Erklärung von Lyons nimmt implizit auf die Quantitätsmaxime von Grice Bezug, die aus den beiden Untermaximen „Mache Deinen Beitrag so informativ wie nötig“ und „Mache Deinen Beitrag nicht informativer als nötig“ besteht (vgl. Grice 1975, S. 45): Da Äußerungen wie $X$ ist nicht gut standardmäßig als ,X ist schlecht" verstanden werden, kann man davon ausgehen, dass der Sprecher mehr gesagt bzw. seine Äußerung präzisiert hätte, wenn er sich nicht auf die Implikatur ,X ist schlecht' hätte festlegen wollen. 
Ähnlich wie Lyons erklärt Levinson die generalisierte konversationale Implikatur ,X ist schlecht' aus X ist nicht gut mit Bezug auf die Quantitätsmaxime von Grice. Nach Levinson ist die Implikatur , schlecht ${ }^{\star}$ aus nicht gut ein Beispiel einer I-Implikatur, d.h. einer Implikatur, die auf dem „Informativeness Principle“, dem I-Prinzip, beruht (Levinson 2000, S. 127-128; zum I-Prinzip vgl. auch Atlas/Levinson 1981, S. 40-41). Das I-Prinzip bezieht sich auf die 2. Quantitätsmaxime von Grice (,Mache Deinen Beitrag nicht informativer als nötig"), aber unterscheidet sich darin von dieser, dass es den Status eines heuristischen Prinzips hat: Es schränkt das Spektrum der möglichen Interpretationen dessen, was mit einer Äußerung kodiert ist, ein. Levinson formuliert das I-Prinzip zunächst nur aus der Perspektive des Adressaten („Was einfach gesagt ist, ist stereotypisch repräsentiert", vgl. Levinson 2000, S. 32) und präzisiert es dann als aus zwei Teilen bestehend, nämlich aus einer Minimisierungsmaxime, an die sich der Sprecher halten soll, und einer Anreicherungsregel für den Adressaten (vgl. Levinson 2000, S. 114):

I-Prinzip

Sprechermaxime (Minimisierungsmaxime): „Sage so wenig wie nötig““

Zusatz für den Adressaten (Anreicherungsregel): „Verstärke den informativen Gehalt der Äußerung des Sprechers, indem Du die spezifischste Interpretation findest, von der Du glaubst, dass sie der kommunikativen Intention des Sprechers entspricht, außer wenn der Sprecher die Minimisierungsmaxime durch den Gebrauch eines markierten oder weitschweifigen Ausdrucks verletzt hat.“

Für den Adressaten lizensiert das I-Prinzip die Implikatur , $\mathrm{X}$ ist schlecht' aus $X$ ist nicht gut, indem es die schwächere Interpretation , $\mathrm{X}$ ist weder gut noch schlecht' ausschließt: Die Tatsache, dass der Sprecher seine Äußerung nicht präzisiert hat, ist für den Adressaten ein Indiz dafür, dass die stereotypische Interpretation gilt.

Der im I-Prinzip enthaltene Verweis auf die Markiertheit sprachlicher Ausdrücke wirft allerdings die Frage auf, ob Äußerungen wie $X$ ist nicht gut im Vergleich zu solchen wie $X$ ist schlecht nicht doch markiert wären. Nach Horn (1989, S. 333) kommt die Implikatur ,schlecht' aus nicht gut gerade dadurch zustande, dass Äußerungen wie Er ist ein schlechter Schachspieler als unhöflich empfunden und deswegen vermieden werden. Horns Erklärung geht auf einen Ansatz von Leech zurück, der die Verwendung negierter Sätze wie I don't like Kenneth, We don't agree und He doesn't believe in marriage als eine Form des „Understatements“ ansieht. Die Negation fungiere in diesen Sätzen als „hedging or mitigating device“, das durch Überlegungen der Höflichkeit und Zurückhaltung beim Ausdrücken von Emotionen und Einstellungen motiviert sei (vgl. Leech 1983, S. 101-102). Äußerungen wie $X$ ist nicht gut sind demnach Euphemismen: Es ist höflicher, jemandem eine gute Eigenschaft abzusprechen als ihm explitzit eine negative zuzuschreiben.

Gegen die Behauptung, dass Äußerungen mit negierten graduierbaren Adjektiven Euphemismen seien, spricht allerdings die Beobachtung, dass negative Verstärkung auch auftritt, wenn Dimensionsadjektive wie gro $\beta$ und hoch, mit denen keine Bewertung ausgedrückt ist, negiert werden (vgl. Levinson 2000, S. 128). Die Verwendung von Äußerungen wie $X$ ist nicht gro $\beta$ als Euphemismen scheint jedenfalls nur in ganz bestimmten Kontexten wie etwa in Situationen, in denen von Besitztümern die Rede ist, möglich. Die Auffassung, 
wonach Äußerungen mit negierten gaduierbaren Adjektiven Euphemismen sind, bietet also keine generelle Erklärung für das Zustandekommen der Standardinterpretation dieser Äußerungen.

\section{4. Äußerungen mit negativ-bewertenden Adjektiven}

Lexikalische Antonyme von positiv-bewertenden Antonymen wie intelligent, also beispielsweise dumm, unterscheiden sich von ihren negierten positiv-bewertenden Pendants wie nicht intelligent in dem, was mit ihnen kodiert ist. Während nicht intelligent alle Bereiche der Skala abdeckt, in denen jemand oder etwas entweder als dumm oder als weder intelligent noch dumm eingeschätzt wird, umfasst der Bereich, der mit dumm kodiert ist, nur den negativen Bereich der Skala. Der mit dumm kodierte Bereich ist also kleiner als der Bereich, der mit nicht intelligent kodiert ist; er deckt sich aber mit dem Bereich, der mit nicht intelligent implikatiert ist (vgl. Abb. 3):

DUMM

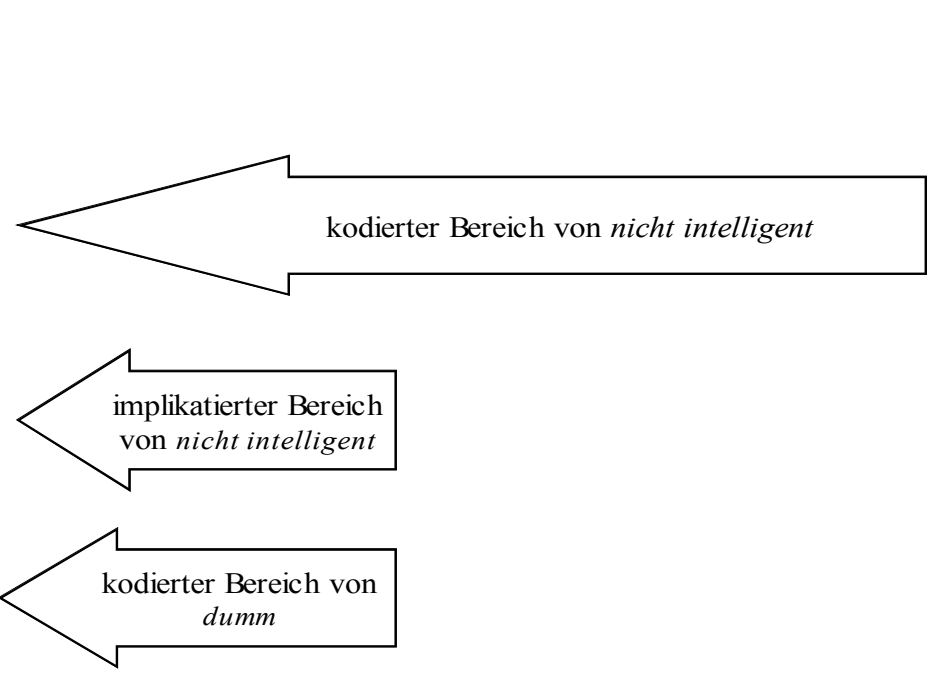

INTELLIGENT

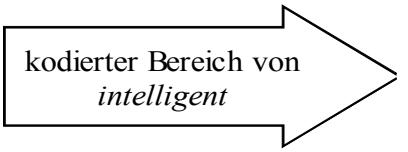

Abb. 3: nicht intelligent vs. dumm

Ähnliches gilt für das Verhältnis von nicht gut und schlecht, nicht froh bzw. nicht glücklich und traurig, nicht spannend und langweilig usw.

Fraglich ist allerdings, ob un-präfigierte Antonyme von positiv-bewertenden Adjektiven die gleiche Bedeutung wie die entsprechenden lexikalischen Antonyme dieser Adjektive haben. Wenn das Präfix un- die Bedeutung, nicht' hat (vgl. Marchand 1969, S. 201; Hofmann 1993, S. 44), sollten negativ-bewertende Adjektive wie unintelligent, unschön, unfroh usw. die Bedeutung, nicht intelligent', ,nicht schön', und ,nicht froh' haben. Der Bereich der relevanten Skala, der mit diesen un-präfigierten Adjektiven jeweils kodiert wäre, würde sich also nicht mit dem Bereich decken, der mit den entsprechenden nichtpräfigierten Formen kodiert ist. Ein Adjektiv wie unintelligent würde also ,nicht intelligent' bedeuten und genauso wie nicht intelligent alle Fälle erfassen, in denen jemand entweder als dumm oder als weder intelligent noch dumm eingeschätzt wird. Ein Adjektiv 
wie dumm könnte hingegen nicht mit Bezug auf den mittleren Bereich der Skala verwendet werden. Wenn unintelligent tatsächlich ,nicht intelligent' bedeutet, wäre es das kontradiktorische Antonym zu intelligent; dumm und intelligent wären hingegen konträre Antonyme (vgl. Abb. 4):
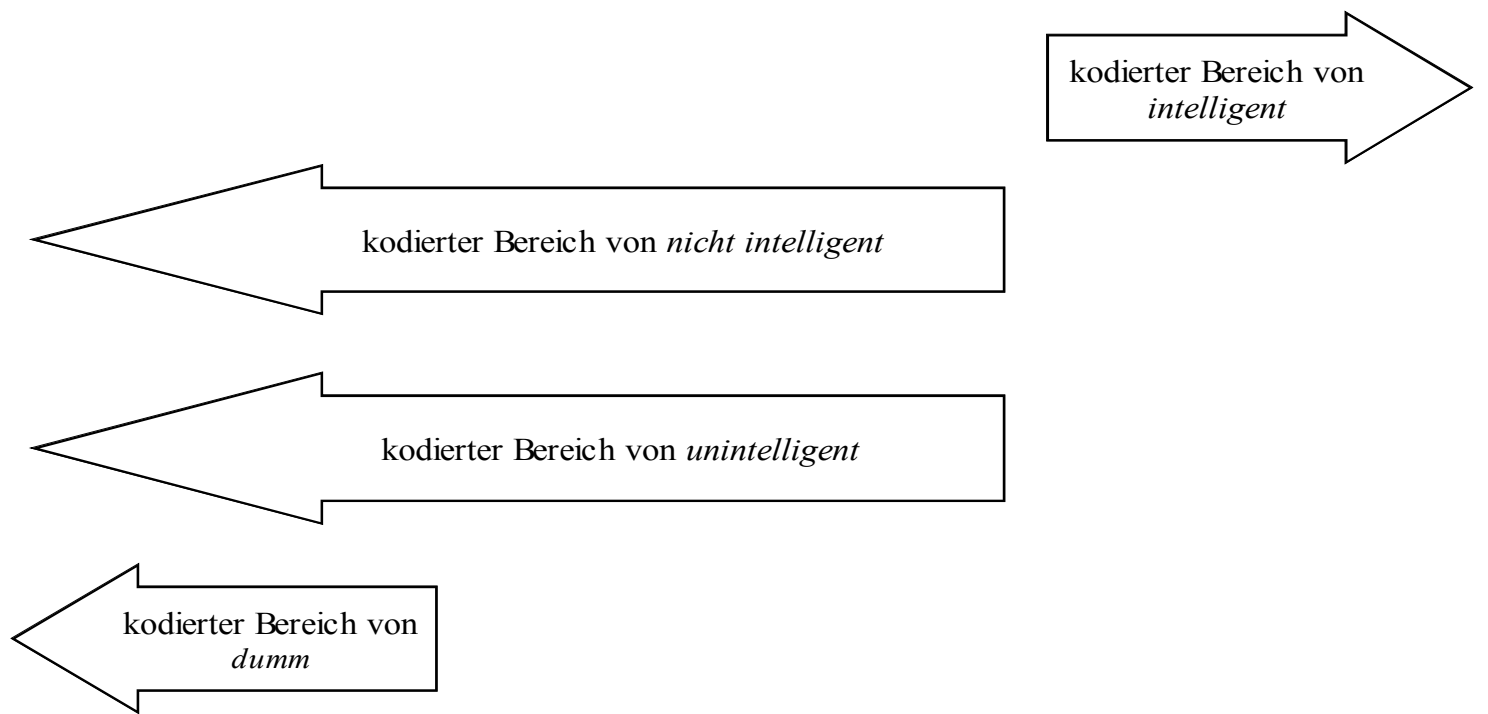

Abb. 4: nicht intelligent/unintelligent vs. dumm

Die Annahme, dass un-präfigierte und lexikalische Antonyme graduierbarer Adjektive sich hinsichtlich des Bereichs der Skala, der mit ihnen jeweils kodiert ist, unterscheiden, stimmt mit der weit verbreiteten Ansicht überein, dass totale Synonymie generell vermieden wird. Nach Kiparsky darf der Output einer lexikalischen Regel beispielsweise nicht synonym mit einer bereits existierenden lexikalischen Einheit sein (vgl. Kiparsky 1983, zitiert in Horn 1989, S. 279).

Wenn un-präfigierte Antonyme positiv-bewertender Adjektive sich tatsächlich darin von den lexikalischen Antonymen dieser Adjektive unterscheiden, dass sie auch mit Bezug auf den mittleren Bereich der Skala angewendet werden können, würde dies bedeuten, dass die beiden Typen von Antonymen in einem Implikationsverhältnis stehen: Wenn jemand dumm ist, ist er auch unintelligent, aber nicht umgekehrt. Die Sätze in (6)-(11) scheinen die Asymmetrie der Implikationsverhältnisse zwischen unintelligent und dumm, ungut und schlecht sowie unschön und hässlich zu bestätigen:

(6a) Seine Reaktion war zwar unintelligent, aber dumm war sie nun auch wieder nicht.

(6b) 'Seine Reaktion war zwar dumm, aber unintelligent war sie nun auch wieder nicht.

(7a) Seine Reaktion war nicht nur unintelligent; sie war sogar dumm. 
(7b) 'Seine Reaktion war nicht nur dumm; sie war sogar unintelligent.

(8a) Die Situation ist zwar ungut, aber schlecht ist sie nun auch wieder nicht.

(8b) 'Die Situation ist zwar schlecht, aber ungut ist sie nun auch wieder nicht.

(9a) Die Situation ist nicht nur ungut; sie ist sogar schlecht.

(9b) 'Die Situation ist nicht nur schlecht; sie ist sogar ungut.

(10a) Die Fassade ist zwar unschön, aber hässlich ist sie nun auch wieder nicht.

(10b) 'Die Fassade ist zwar hässlich, aber unschön ist sie nun auch wieder nicht.

(11a) Die Fassade ist nicht nur unschön; sie ist sogar hässlich.

(11b) 'Die Fassade ist nicht nur hässlich; sie ist sogar unschön.

Die Tatsache, dass die Beispiele in (6b)-(11b) im Unterschied zu denen in (6a)-(11a) nur schwer zu interpretieren sind, zeigt, dass die Implikationsverhältnisse zwischen den unpräfigierten und den lexikalischen Antonymen positiv-bewertender Adjektive asymmetrisch sind. Das ist darauf zurückzuführen, dass Erstere einen größeren Bereich der jeweils relevanten Skala umfassen als Letztere.

Korpusbelege, die Aufschluss über den Intensivierungsgrad von un-präfigierten vs. lexikalischen Antonymen positiv-bewertender Adjektive geben, lassen sich nur für unschön vs. hässlich finden:

(12a) Zugegeben, wir haben unschöne, um nicht zu sagen nachgerade hässliche Szenen in den letzten Tagen erlebt. (die tageszeitung, 13.11.1989, S. 24)

(12b) Weil die betreffenden saubösen Briefeschreiber nicht nur unehrlich, sondern auch fantasielos sind. Aber ,unschöne“ oder gar „, hässliche Grüße“ klingt ja auch blöd. (die tageszeitung, 28.02.2004, S. VIII)

Diese Belege bestätigen, dass unschön zwar zu hässlich gesteigert werden kann, aber nicht umgekehrt: Etwas, das als hässlich beurteilt wird, wird zugleich auch als unschön eingeschätzt, aber etwas, das als unschön angesehen wird, wird nicht notwendigerweise auch als hässlich beurteilt.

Unklar ist aber, ob ein derartiges asymmetrisches Implikationsverhältnis auch für unglücklich und traurig gilt:

(13a) Er ist zwar unglücklich, aber traurig ist er nun auch wieder nicht.

(13b) Er ist zwar traurig, aber unglücklich ist er nun auch wieder nicht.

Dass Satz (13b) eher akteptabel scheint als die Sätze in (6b)-(12b) mag damit zusammenhängen, dass die Verbindung von un- und positiv-bewertendem Adjektiv in unglücklich stärker lexikalisiert ist als in unintelligent oder ungut. Levinson (2000, S. 144) geht zwar nicht explizit auf das Verhältnis von $u n$-präfigierten und lexikalischen Antonymen positivbewertender Adjektive ein, aber die Tatsache, dass er unhappy an die gleiche Position der Skala wie sad einordnet, suggeriert, dass er die beiden Adjektive als bedeutungsgleich ansieht (vgl. Abb. 5). 

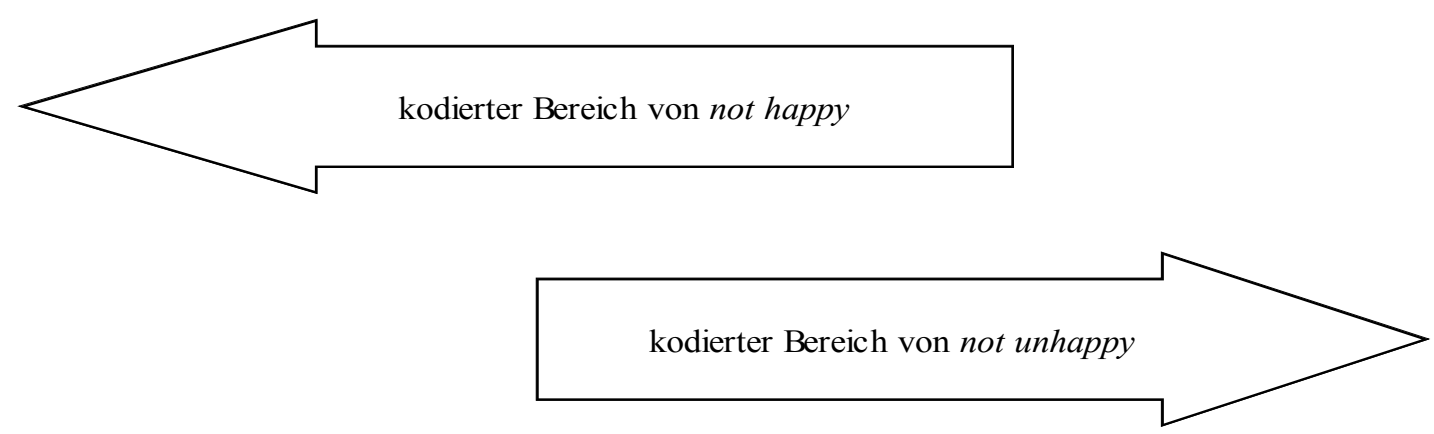

Abb. 5: unhappy-happy-Skala nach Levinson (2000, S. 144)

Die Tatsache, dass es keinen deutlichen Unterschied in der Akzeptabilität von (13a) und (13b) gibt, könnte aber auch darauf zurückzuführen sein, dass unglücklich und traurig bzw. unhappy und sad keine entgegengesetzten Bereiche ein und derselben Skala, sondern vielmehr je einen Teilbereich unterschiedlicher Kontinua bezeichnen. Lehrer weist beispielsweise darauf hin, dass morphologhische, d.h. un-präfigierte Antonyme wie unhappy meist eine allgemeinere Bedeutung als die entsprechenden lexikalischen Antonyme (in diesem Fall $s a d$ ) haben. Unter dem Hyperonym unhappy könnten beispielsweise die Adjektive sad, angry und depressed subsumiert werden, mit denen auf jeweils unterschiedliche Skalen Bezug genommen wird (vgl. Lehrer 2002, S. 504). Im Deutschen kämen Adjektive wie deprimiert, depressiv, verzweifelt und eben auch traurig als Hyponyme von unglücklich in Frage. Wenn traurig tatsächlich ein Hyponym von unglücklich wäre, könnte das der Grund dafür sein, dass der Satz in (14b) weniger akzeptabel erscheint als der in (14a):

(14a) Er ist unglücklich, aber nicht traurig.

(14b) 'Er ist traurig, aber nicht unglücklich.

Wenn die Bedeutung von traurig in der Bedeutung von unglücklich enthalten ist, ist ein Satz wie der in (14b) widersprüchlich und damit schwer zu interpretieren.

Zur Bedeutung von $u n$-präfigierten vs. lexikalischen Antonymen graduierbarer Adjektive sind in der Literatur unterschiedliche Positionen vertreten worden. Nach Zimmer (1964, S. 87-88) können durch un-Präfigierung im Englischen und im Deutschen sowie durch in-Präfigierung im Französischen sowohl kontradiktorische als auch konträre Antonyme gebildet werden. Konträre Antonyme wie etwa unhappy als Antonym zu happy seien „ungeneriert" (,ungenerated“), d.h. lexikalisierte Verbindungen von Morphemen, während kontradiktorische Antonyme wie unopenable als Antonym zu openable generierte, d.h. produktive Morphemkombinationen seien. Auch Hofmann (1993, S. 44) vertritt die Meinung, dass durch die un-Präfigierung graduierbarer Adjektive negative Skalen gebildet werden, die vom Mittelbereich ausgehen. Beide Ansichten stimmen mit der hier ver- 
tretenen Auffassung überein, dass durch die un-Präfigierung graduierbarer Adjektive in der Regel kontradiktorische Antonyme gebildet werden und konträre Antonyme wie unglücklich lexikalisierte Kombinationen von un- und Adjektiv seien.

Lehrer (2002, S. 504) hingegen führt nicht nur happy-unhappy, sondern auch intelligentunintelligent und wise-unwise als Beispiele konträrer Antonyme auf. Das scheint zunächst plausibel, weil man von jemandem behaupten kann, dass er weder intelligent noch unintelligent bzw. weder glücklich noch unglücklich ist, ohne dass dies zu einem Widerspruch führt. Diese Beobachtung kann einerseits als Indiz dafür gewertet werden, dass intelligent und unintelligent entgegengesetzte Bereiche einer Skala bezeichnen, die auch einen Mittelbereich enthält, was dafür sprechen würde, diese Adjektive tatsächlich als konträre Antonyme anzusehen. Andererseits könnte die Nicht-Widersprüchlichkeit von Äußerungen wie Er ist weder intelligent noch unintelligent auch darauf zurückzuführen sein, dass unintelligent aus logischer Sicht zwar, nicht intelligent' bedeutet, aber üblicherweise als das konträre Antonym dieses Adjektivs verwendet wird. , $X$ ist dumm' wäre damit die Implikatur aus Äußerungen wie $X$ ist unintelligent (vgl. Abb. 6). Wenn unintelligent tatsächlich ,nicht intelligent' bedeutet, scheint diese Erklärung durchaus plausibel: Was für nicht intelligent gilt, müsste auch für unintelligent gelten. Das entspricht auch der Meinung, die Jespersen bereits 1917 vertreten hat:

The modification in sense brought about by the addition of the prefix [un-] is generally that of a simple negation: unworthy $=$, not worthy', etc. ... The two terms $[X, u n X]$ are thus contradictory terms. But very often the prefix produces a ,contrary' term or at any rate what approaches one: unjust generally implies the opposite of just; unwise means more than not wise and approaches foolish, unhappy ist not far from miserable, etc. (Jespersen 1942, S. 466-467)

DUMM
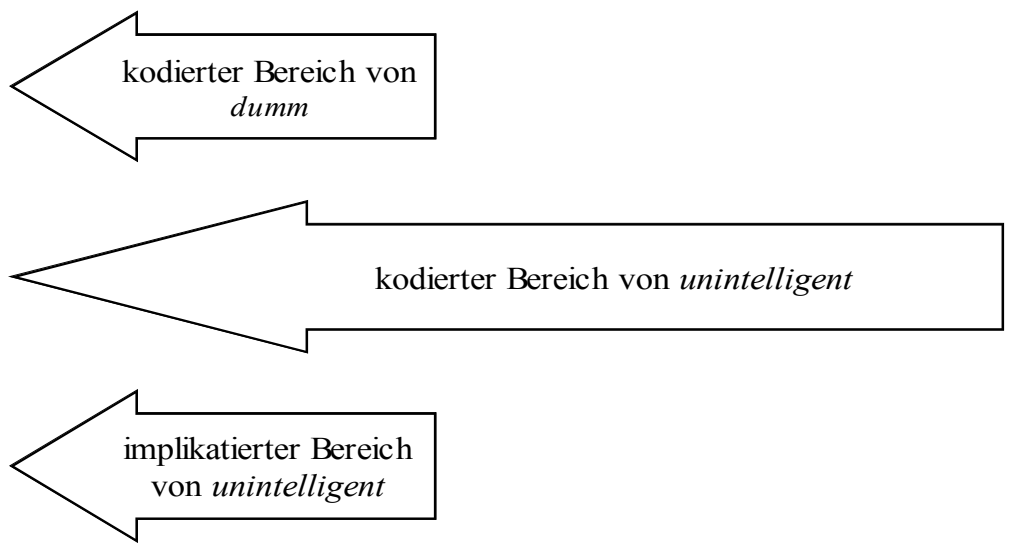

Abb. 6: unintelligent: kodierter und implikatierter Bereich
INTELLIGENT

Die Erklärung dafür, dass un-präfigierte Adjektive wie unintelligent als konträre Antonyme ihres Grundworts verwendet werden, obwohl sie logisch geschehen das kontradiktorische Antonym dieses Grundworts sind, könnte auch in diesem Fall darin bestehen, dass die un-präfigierten Formen als Euphemismen fungieren. Die Verwendung von unin- 
tellingent anstelle von dumm wäre demnach ein weiteres Beispiel von „contraries in contradictory cloathing“" (vgl. Horn 1989, S. 273): Gemeint ist ,X ist dumm“, aber gesagt wird nur, dass X unintelligent ist. Wie auch nicht intelligent fungiert unintelligent hier als ,Tarnung“, Horns „cloathing“, für dumm. Wenn un-präfigierte Formen wie unintelligent tatsächlich als Euphemismen verwendet werden, ist es nicht verwunderlich, dass viele der Adjektive, die sowohl ein un-präfigiertes als auch ein morphologisch nicht-verwandtes Antonym haben, evaluative Adjektive sind.

Obwohl auch manche nicht-bewertenden Adjektive sowohl ein lexikalisches als auch ein un-präfigiertes Antonym haben, unterscheiden sich diese zumindest in manchen Fällen grundlegend in ihrer lexikalischen Bedeutung. Ein Beispiel ist gesund, für das es sowohl das Antonym krank als auch das Antonym ungesund gibt. Diese unterscheiden sich jedoch in ihrer lexikalischen Bedeutung: Während die Bedeutung von krank als ,Zeichen von Krankheit aufweisend" paraphrasiert werden kann, hat ungesund die Bedeutungen ,schlecht für die Gesundheit‘ (vgl. Bsp. (15)), ,krank aussehend‘ (vgl. Bsp. (16)) und , unvernünftig'(vgl. Bsp. (17)).

(15) Gefrierbrand mindert zwar die Qualität der Ware, ist aber nicht gesundheitsschädlich. Richtig ungesund sind dagegen Krankheitserreger auf Schnitzel oder Keule. (Die Tageszeitung, 02.10.1993, S. 31; „Für Fachleute nichts Neues“)

(16) Wir verbringen den Tag mit dem Verfassen von Abschiedsbriefen, ..., pflegen unsere ungesunde Gesichtsfarbe mit einem übermäßigen Zigarettenkonsum, ... (Frankfurter Rundschau, 11.01.1997, S. 6, Ressort: Zeit und Bild)

(17) Kommen für solche Schleudersitze nicht vor allem ... die gierigsten Egozentriker in Frage, ..., Persönlichkeiten, die in der Vereinsamung, die die Machtausübung im Grunde fast immer mit sich bringt, dazu neigen (müssen?), sich in ungesunde Richtungen zu verändern, bis hin zu Verfolgungs- oder Größenwahn oder etwa zur Kleptomanie? (Tiroler Tageszeitung, 18.12.1998; Ressort: Tirol aktuell; Gelegenheit macht Diebe auch aus Präsidenten?)

Damit wäre ungesund ein negativ-bewertendes Adjektiv und krank ein nicht-bewertendes Zustandsadjektiv, was Aufschluss über die Polysemie von gesund gibt: Die Antonymie von gesund und krank betrifft die Bedeutung von gesund als Zustandsprädikat (,keine Krankheitszeichen aufweisend'); die von gesund und ungesund die Bedeutung von gesund als Bewertungsprädikat (,durch sein Aussehen von Gesundheit zeugend'bzw. , gut für die Gesundheit'). Die gelegentliche Verwendung von krank als Bewertungsadjektiv, die sich beispielsweise in Äußerungen wie Die Idee ist echt krank zeigt, kann als eine metaphorische Erweiterung der Bedeutung von krank als Zustandsadjektiv aufgefasst werden, die sowohl Verwendungen mit Bezug auf physische als auch Verwendungen mit Bezug auf auf psychische Krankheiten mit einschließt. Da die Antonyme krank und ungesund jeweils auf eine andere Bedeutung von gesund zugreifen, kann ungesund nicht als Euphemismus für krank verwendet werden.

un-präfigierte und lexikalische Antonyme graduierbarer Adjektive unterscheiden sich manchmal auch hinsichtlich der Kontexte, in denen sie typischerweise verwendet werden. Adjektive wie ungut und unschön werden z.B. häufig mit Bezug auf Situationen verwendet, in denen menschliche Verhaltensweisen oder deren Resultate, zu denen auch 
Artefakte gehören, beurteilt werden. Zur Bezugnahme auf diese Situationen bieten sich euphemistische Ausdrücke wie ungut und unschön eher als schlecht bzw. hässlich an. Die Korpusbelege in (18)-(20) zeigen typische Verwendungsweisen von ungut und unschön:

(18) Von unguten Erfahrungen mit der Braunauer Firma in München weiß eine frühere Bestellerin von „Dianetik“: Wenn die einmal deine Adresse haben, wirst du mit Telefonanrufen bombardiert. (Tiroler Tageszeitung, 30.10.1996)

(19) Jeder, der ein altes, „unmodernes und unschönes“ Regal mitbringt, kann es gegen ein neues umtauschen - solange Vorrat reicht, ... (die tageszeitung, 01.10.1999, S. 23)

(20) „Das Ende war unschön und kein Fussball mehr“, räumte denn auch Stefan Beinlich, der Leverkusener Torschütze zum 1:0, ein. (Züricher Tagesanzeiger, 11.12.1997, S. 55)

Die lexikalischen Pendants von ungut und unschön, schlecht und hässlich, werden weitaus häufiger als ungut und unschön mit Bezug auf natürliche Zustände und Ereignisse verwendet, in denen der Bedarf an abschwächenden Ausdrücken viel geringer ist. Für die Verwendung von ungut und unschön zur Beschreibung von rein natürlichen Phänomenen wie etwa einem Baum, einer Landschaft oder dem Wetter ließen sich in den IDS-Korpora jedenfalls keine Belege finden.

Die Beobachtungen zur Verwendung von un-präfigierten vs. lexikalischen Antonymen graduierbarer Adjektive zeigen, dass Erstere im Gegensatz zu Letzteren einen anderen Bezugsbereich als das jeweilige nicht-präfigierte Pendant haben. Das bedeutet, dass unpräfigierte Antonyme keine Dubletten der entsprechenden lexikalischen Antonyme oder der negierten Form ihrer nicht-präfigierten Pendants (nicht $X$ ) sind.

\section{5. Äußerungen mit negierten negativ-bewertenden Adjektiven}

Da lexikalische Antonyme positiv-bewertender Adjektive wie intelligent, gut und schön, also beispielweise dumm, schlecht und hässlich, nur mit Bezug auf den negativen Bereich der jeweiligen Skala verwendet werden können, sind mit den negierten Formen dieser Adjektive jeweils nur der mittlere und der positive Bereich der Skala kodiert. Wenn die un-präfigierten Pendants dieser lexikalischen Antonyme, also beispielsweise unintelligent, ungut und unschön sowohl mit Bezug auf den mittleren als auch mit Bezug auf den negativen Bereich der jeweils relevanten Skala verwendet werden können, ist mit den negierten Formen dieser un-präfigierten Antonyme nur der positive Bereich der Skala kodiert. Äußerungen wie $X$ ist nicht unintelligent haben demnach die Bedeutung, $\mathrm{X}$ ist intelligent', d.h. die doppelte Negation (nicht un-) ergibt eine positive Interpretation (vgl. Abb. 7). 


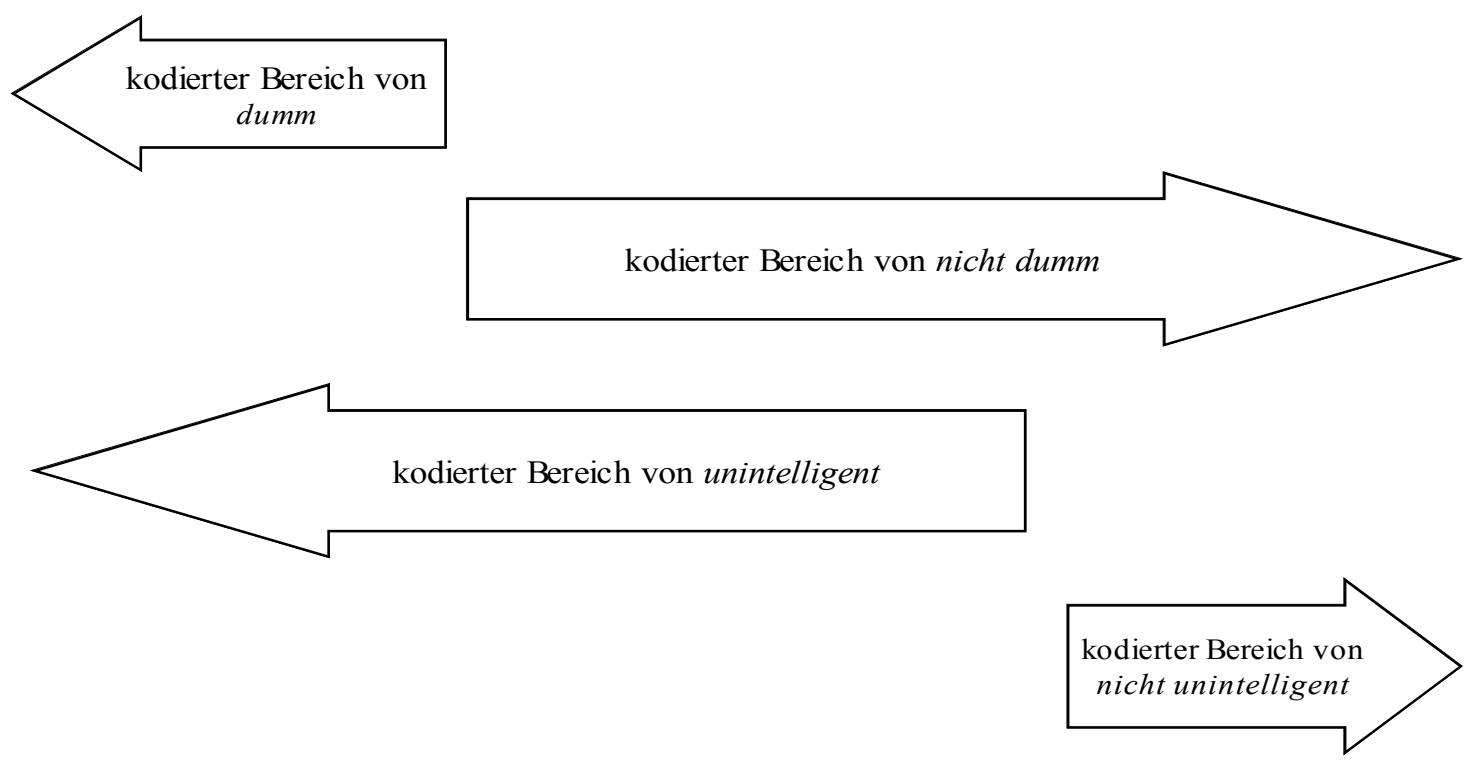

Abb. 7: nicht dumm vs. nicht unintelligent: kodierter Bereich

Obwohl nicht dumm logisch sowohl mit dem Zustand des Intelligent-Seins als auch mit dem, in dem jemand weder dumm noch intelligent ist, kompatibel ist, werden Äußerungen wie Er ist nicht dumm üblicherweise so interpretiert, dass die Person, von der die Rede ist, als eher intelligent oder ziemlich intelligent beurteilt wird. Ähnlich werden Äußerungen wie Er ist nicht unintelligent normalerweise nicht so verstanden, dass die betreffende Person intelligent ist, sondern vielmehr so, dass sie eher oder ziemlich intelligent ist. Beide Typen von Äußerungen werden also so interpretiert, dass die Person, von der die Rede ist, zwar intelligent, aber auch nicht ganz so intelligent ist, wie der Sprecher durch die Verwendung von intelligent zu verstehen gegeben hätte. Die Standardinterpretation ,X ist eher/ziemlich intelligent" von Äußerungen wie $X$ ist nicht unintelligent oder $X$ ist nicht dumm schließt die Interpretation, sehr intelligent' aus.

Die Verwendung von doppelt negierten Ausdrücken wie nicht unintelligent mit Bezug auf Situationen, in denen jemand als eher oder ziemlich intelligent beurteilt wird, ist ein Beispiel einer rhetorischen Figur, die als „Litotes“ bezeichnet wird: nicht unintelligent wird nicht als ,intelligent' interpretiert, d.h. der doppelt negierte Ausdruck erhält in diesem Fall keine positive Interpretation (vgl. Horn 1989, S. 296-308; Hofmann 1993, S. 50-52; Levinson 2000, S. 142; Blutner 2004, S. 499-501). Levinson generalisiert das folgendermaßen: „Wenn W eine positiv-bewertende lexikalische Einheit ist, und W“ ist das konträre Antonym von W, dann ist $\neg\left(\mathrm{W}^{6}\right)$ nicht äquivalent mit W“ (Levinson 2000, S. 143). Die pragmatische Leistung der Litotes besteht darin, dass sie es ermöglicht, andere Bereiche der Skala zu verstehen zu geben als die, die mit den entsprechenden Ausdrücken direkt ausgedrückt bzw. kodiert oder gesagt sind. 
Die Standardinterpretation , $\mathrm{X}$ ist eher/ziemlich intelligent' von Äußerungen wie $X$ ist nicht unintelligent ist nach Levinson ein Beispiel einer M-Implikatur, d.h. einer Implikatur, die auf dem M-Prinzip („Manner Principle“) basiert. Das von Levinson formulierte M-Prinzip geht auf Grices Modalitätsmaxime zurück, die aus der übergeordneten Maxime „Sei klar“ und den folgenden vier Submaximen besteht: (i) „Vermeide Dunkelheit des Ausdrucks“, (ii) „Vermeide Mehrdeutigkeiten“, (iii) „Sei kurz“ bzw. „Vermeide unnötige Weitschweifigkeit" und (iv) „Gehe der Reihe nach vor“" bzw. „Sei folgerichtig“ (vgl. Grice 1975, S. 46). Levinsons M-Prinzip umfasst nur die erste und den formalen Aspekt der dritten von Grices Submaximen. Die anderen der von Grice formulierten Submaximen schließt Levinson aus seinem M-Prinzip aus mit der Begründung, dass (i) die zweite Submaxime („Vermeide Mehrdeutigkeiten“) teilweise duch das Q-Prinzip abgedeckt sei (,Was nicht gesagt ist, ist nicht", vgl. Levinson 2000, S. 35) und Ambiguität generell kaum zu vermeiden sei, (ii) der Informationsaspekt von Grices dritten Submaxime (,Vermeide Dunkelheit des Ausdrucks“) bereits in der Minimisierungsmaxime des I-Prinzips enthalten sei („Sage so wenig wie nötig“) und (iii) Grices vierte Submaxime (,Sei folgerichtig“) nicht den Status einer Maxime habe, sondern vielmehr eine natürliche kognitive Neigung reflektiere, die sich aus dem Zusammenspiel der Ikonizität der Sprache und der Linearität des Sprechens ergebe (vgl. Levinson 2000, S. 135-136).

Ähnlich wie das I-Prinzip formuliert Levinson das M-Prinzip zunächst als ein heuristisches Prinzip („Was in einer nicht-normalen Weise gesagt ist, ist nicht normal“, (Levinson 2000, S. 38)), das dann folgendermaßen präzisiert wird (Levinson 2000, S. 136-137):

\section{M-Prinzip}

Sprechermaxime: „Verwende für die Bezugnahme auf nicht-normale, nicht-stereotypische Situationen markierte Ausdrücke, die einen Kontrast mit denjenigen Ausdrücken bilden, die Du zur Bezugnahme auf die entsprechende normale, stereotypische Situation verwenden würdest.“

Zusatz für den Adressaten: Was auf nicht-normale Art und Weise gesagt ist, bezeichnet eine nicht-normale Situation; markierte Ausdrücke bezeichnen markierte Situationen.

Das M-Prinzip erklärt, warum Äußerungen wie $X$ ist nicht unintelligent oder $X$ ist nicht dumm nicht als ,X ist intelligent' interpretiert werden: Der Adressat weiß, dass der Sprecher den unmarkierten Ausdruck (hier intelligent) gewählt hätte, wenn dieser die Person, von der die Rede ist, als intelligent einschätzen würde. Da der Sprecher aber einen anderen, markierten Ausdruck (hier nicht unintelligent) gewählt hat, nimmt der Adressat an, dass der Sprecher etwas anderes als ,intelligent' gemeint haben muss. Aus dieser Überlegung schließt er, dass die betreffende Person zwar intelligent, aber auch nicht ganz so intelligent ist, wie der Sprecher durch die Wahl von intelligent zu verstehen gegeben hätte.

\section{6. Äußerungen mit negierten un-präfigierten bzw. mit negierten lexikalischen Antonymen positiv-bewertender Adjektive}

Da negierte Adjektive wie nicht dumm und nicht unintelligent beide als ,eher intelligent ${ }^{*}$ oder, ziemlich intelligent' interpretiert werden, erhebt sich die Frage, ob sich die beiden Ausdrücke hinsichtlich des mit ihnen implikatierten Bereichs der Skala unterscheiden. Muttersprachler scheinen dazu zu tendieren, die mit den negierten un-präfigierten Formen 
ausgedrückte Bewertung als positiver zu beurteilen als die Bewertung, die mit den negierten nicht-abgeleiteten Formen ausgedrückt ist. Der mit nicht dumm implikatierte Bereich wäre demnach weiter vom extrem positiven Pol der Skala entfernt als der Bereich, der mit nicht unintelligent implikatiert ist (vgl. Abb. 8).

DUMM

WEDER INTELLIGENT NOCH DUMM

INTELLIGENT

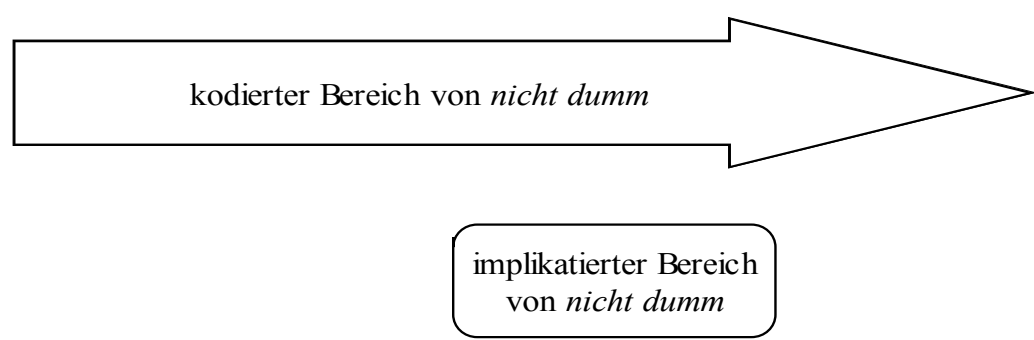

Abb. 8: nicht dumm vs. nicht unintelligent: kodierter und implikatierter Bereich

Die Beobachtung, dass die mit den negierten, un-präfigierten Formen ausgedrückte Bewertung häufig als positiver eingeschätzt wird als die Bewertung, die mit den negierten nicht-abgeleiteten Formen ausgedrückt ist, basiert auf Kompetenzurteilen von einer kleinen Anzahl von Muttersprachlern, die natürlich in keinerlei Weise erschöpfend sind. Wünschenswert wäre, wenn diese Kompetenzurteile empirisch überprüft werden könnten. Eine Möglichkeit, Kompetenzurteile zu den Implikaten von Äußerungen mit Ausdrücken wie nicht dumm bzw. von solchen mit nicht unintelligent empirisch zu überprüfen, könnte darin bestehen, in einem Korpus gezielt nach Belegen zu suchen, in denen die Ausdrücke, deren Intensivierungsgrad verglichen werden soll, zusammen vorkommen, wie etwa in Beispiel (13a) (Zugegeben, wir haben unschöne, um nicht zu sagen nachgerade häßliche Szenen in den letzten Tagen erlebt). Die Suche nach Korpusbelegen, in denen negierte Ausdrücke wie nicht unschön und nicht hässlich im gleichen Kontext vorkommen, ergab aber keine Treffer.

Auch das Ersetzen vom einen durch den anderen Ausdruck in Beispielsätzen erweist sich als unbrauchbar: Die negierten un-präfigierten Formen können fast immer durch die entsprechenden negierten nicht-abgeleiteten Formen ersetzt werden, ohne dass dies irgendetwas über den Intensivierungsgrad der beiden Ausdrücke aussagt:

(21) Die ja durchaus nicht langweilige [nicht unspannende] Geschichte Kretas wird auf ganzen fünf Seiten abgehandelt. (Die Tageszeitung 1992)

(22) Das Thema ist nicht unspannend [nicht langweilig]. 
Eine andere Möglichkeit, die Kompetenzurteile von Sprechern zu den Implikaturen von Äußerungen mit Ausdrücken wie nicht dumm bzw. nicht unintelligent empirisch zu überprüfen, wäre Sprecher gezielt zu ihren Intuitionen zu befragen. Das könnte man z.B. tun, indem man Sprecher die (In)Akzeptabilität von Sätzen mit den entsprechenden negativen Prädikaten beurteilen lässt. Mögliche Beispiele solcher Sätze wären:

(23) Das Thema ist nicht langweilig; man könnte sogar sagen, es ist nicht unspannend. vs.

(24) Das Thema ist nicht unspannend; man könnte sogar sagen, es ist nicht langweilig.

Eine andere Möglichkeit wäre, Sprechern Geschichten vorzulegen und ihnen negierte Prädikate zur Auswahl zu stellen, um diese zu beschreiben (z.B. nicht intelligent - nicht dumm - nicht unintelligent).

\section{7. $\quad$ Fazit und Ausblick}

Äußerungen wie $X$ ist nicht dumm und $X$ ist nicht unintelligent unterscheiden sich sowohl im Hinblick auf das, was mit ihnen kodiert ist, als auch in Bezug auf das, was mit ihnen implikatiert ist. Dass die beiden Typen von Äußerungen sich in dem, was mit ihnen kodiert ist, unterscheiden, ist darauf zurückzuführen, dass die nicht-negierten Formen dieser Äußerungen, $X$ ist dumm bzw. $X$ ist unintelligent ebenfalls einen unterschiedlichen Bereich der relevanten Skala kodieren: Während dumm nur mit Bezug auf den negativen Bereich der Skala verwendet wird, ist mit unintelligent sowohl der negative als auch der neutrale Mittelbereich kodiert. Dementsprechend unterscheiden sich auch die negierten Formen von dumm und unintelligent im Hinblick auf das, was mit ihnen kodiert ist: nicht dumm umfasst sowohl den positiven als auch den neutralen Mittelbereich der Skala, nicht unintelligent hingegen nur den positiven Bereich.

nicht dumm und nicht unintelligent unterscheiden sich aber auch hinsichtlich ihrer Standardinterpretation: Obwohl beide Ausdrücke als ,eher intelligent' oder ,ziemlich intelligent" interpretiert werden, unterscheiden sie sich dennoch im Grad der mit ihnen ausgedrückten positiven Bewertung. Auch in Fällen, in denen für ein bestimmtes positivbewertendes Adjektiv nur eine Form mit gegensätzlicher Bedeutung zur Verfügung steht, wird das negierte negativ-bewertende Adjektiv (nicht $X$ ) als , eher $\mathrm{X}^{6}$ oder , ziemlich $\mathrm{X}^{6}$ interpretiert. Äußerungen wie Sie ist nicht faul und Er ist nicht unzufrieden, werden beispielsweise so interpretiert, dass die Person, von der die Rede ist, eher fleißig bzw. zufrieden ist. Wenn sowohl eine negierte un-präfigierte als auch eine negierte nicht-abgeleitete Form zur Verfügung stehen, wird die Bewertung, die mit der negierten un-präfigierten Form ausgedrückt wird, als positiver beurteilt als die Bewertung, die mit der negierten nicht-abgeleiteten Form zum Ausdruck gebracht wird. Allerdings müsste noch empirisch überprüft werden, ob solche subtilen Unterschiede in Bezug auf den mit beiden Formen implikatierten Bereich sich auch für eine repräsentative Menge von Muttersprachlern bestätigen lassen. Noch zu klären wäre außerdem, ob es auch andere als evaluative Adjektive gibt, für die zwei Formen mit gegensätzlicher Bedeutung zur Verfügung stehen. Wenn dies tatsächlich der Fall wäre, könnte das Vorhandensein eines un-präfigierten neben einem lexikalischen Antonym nicht generell mit dem Bedarf an Euphemismen erklärt werden. 


\section{Literatur}

Atlas, Jay D./Levinson, Stephen C. (1981): It-Clefts, Informativeness, and Logical Form: Radical Pragmatics (Revised Standard Version). In: Cole, Peter (Hg.): Radical Pragmatics. New York. S. 1- 61.

Blutner, Reinhard (2004): Pragmatics and the Lexicon. In: Horn, Laurence R./Ward, Gregory (Hg.): The Handbook of Pragmatics. Oxford. (Blackwell Handbooks in Linguistics 16). S. 488-514.

Cruse, D. Alan (1986): Lexical Semantics. Cambridge.

Grice, Herbert P. (1975): Logic and Conversation. In: Cole, Peter/Morgan, Jerry L. (Hg.): Syntax and Semantics. Vol. 3: Speech Acts. New York. S. 41-58.

Harras, Gisela (2003): Konvention oder Kooperation? Das Fundament der diskursiven Sinnfindung. In: Hagemann, Jörg/Sager, Sven F. (Hg.): Schriftliche und mündliche Kommunikation: Begriffe - Methoden - Analysen. Festschrift zum 65. Geburtstag von Klaus Brinker. Tübingen. S. 49-60.

Harras, Gisela (2004): Handlungssprache und Sprechhandlung: Eine Einführung in die theoretischen Grundlagen. 2. Auflage. Berlin. (de Gruyter Studienbuch).

Hofmann, Thomas R. (1993): Realms of Meaning: An Introduction to Semantics. London. (Learning About Language).

Horn, Laurence R. (1989): A Natural History of Negation. Chicago.

Horn, Laurence R. (2004): Implicature. In: Horn, Laurence R./Ward, Gregory (Hg.): The Handbook of Pragmatics. Oxford. (Blackwell Handbooks in Linguistics 16). S. 3-28.

Jespersen, Otto (1942): A Modern English Grammar on Historical Principles. Part VI: Morphology.

Leech, Geoffrey (1983): Principles of Pragmatics. London. (Longman Linguistics Library 30).

Lehrer, Adrienne J. (2002): Paradigmatic relations of exclusion and opposition I: Gradable antonymy and complementarity. In: Cruse, D. Alan/Hundsnurscher, Franz/Job, Michael/Lutzeier, Peter R. (Hg.): Lexikologie: Ein internationales Handbuch zur Natur und Struktur von Wörtern und Wortschätzen. 1. Halbband. Berlin. S. 498-507.

Levinson, Stephen C. (2000): Presumptive Meanings: The Theory of Generalized Conversational Implicature. Cambridge/MA.

Löbner, Sebastian (2003): Semantik: Eine Einführung. Berlin. (de Gruyter Studienbuch).

Lyons, John (1977): Semantics. Bd. I. Cambridge.

Marchand, Hans (1969): The Categories and Types of Present-Day English Word-Formation: A SynchronicDiachronic Approach. 2. Auflage. München.

Rachidi, Renate (1989): Gegensatzrelationen im Bereich deutscher Adjektive. Tübingen. (Reihe Germanistische Linguistik 98).

Zimmer, Karl (1964): Affixal Negation in English and other Languages: An Investigation of Restricted Productivity. Supplement zu WORD 20, 2.

Dr. Kristel Proost

Institut für Deutsche Sprache

Postfach 101621

D-68016 Mannheim

e-mail:proost@ids-mannheim.de 